\title{
Validation d'un modèle de durée de développement larvaire de la pyrale du maïs, Ostrinia nubilalis Hbn, Lepidoptera, Pyralidae, en France
}

\author{
B Got, GF Lacan, N Smits, E Stephan \\ INRA, station de zoologie, route de Saint-Cyr, 78026 Versailles, cedex, France
}

(Reçu le 27 mars 1990; accepté le 15 novembre 1990)

\begin{abstract}
Résumé - Cet article présente la validation d'un modèle de durée de développement larvaire de la pyrale du maïs sur plusieurs variétés de maïs et dans 4 sites géographiques en France : Avignon, Bordeaux, Colmar et Versailles. Ce modèle est composé d'un modèle de degrés-jours et de lois normales. Il a été estimé et testé sur LG11 en région parisienne. Les validations montrent que le modèle représente correctement le développement à moins d'une semaine près. Il est donc satisfaisant pour des objectifs agronomiques (prévision des diminutions de rendement, lutte contre l'insecte). Les possibilités d'amélioration sont discutées : compte tenu des connaissances acquises sur le ravageur, l'utilisation d'une relation non linéaire pour représenter la liaison entre vitesse de développement et température et la prise en compte de la température au niveau de la végétation semblent être les voies d'amélioration de la précision du modèle les plus réalistes.
\end{abstract}

modèle mathématique / développement larvaire / validation / Ostrinia nubilalis = pyrale du maïs / insectes

Summary - Validation of a model for European corn borer development in France. The validation of a temperature-dependent model for European Corn Borer development on 3 different corn varieties (LG11, DEA and ISORA), at 4 sites in France : Avignon, Bordeaux, Colmar and Versailles, and with 2 infestation times for each site and variety, is presented. The model consist of 2 parts: a degree-day model and 4 normal distributions (fig 1). It has been estimated and tested at Versailles (table II). The validations show that the mean differences between predicted and observed data are systematically less than a week under the different experimental conditions (tables III and IV, figs $3 a, 3 b, 4$ and 5). The model is then an appropriate representation of development for agronomical purposes (prediction of losses, pest control). Possible improvements of the model are discussed: using a non linear model between growth rate and temperature and taking into account the temperature under vegetation seem to be the most realistic ways to improve the model, considering the knowledge of European corn borer biology.

mathematical model / larval development time / validation / Ostrinia nubilalis = European corn borer / insecta

\section{INTRODUCTION}

La pyrale du maïs, Ostrinia nubilalis $\mathrm{Hbn}$, est le principal ravageur du maïs dans de nombreux pays d'Europe, d'Amérique et d'Asie. Les dégats provoqués par les stades larvaires de l'insecte sont fonction de l'importance de l'infestation, du stade du maïs lors de la ponte, de l'emplacement et de la date de réalisation des dégats (Stengel, 1969; Lynch, 1980; Lynch et al, 1980). De ce fait, les régressions reliant les pertes aux nombres de pontes, de chenilles ou de dégâts n'ont pas permis d'expliquer correctement la diminution de rendement (Sparks et al, 1967) et montrent la nécessité d'une meilleure prédiction des dégâts. C'est dans le but de comprendre et de quantifier l'ensemble des phénomènes depuis l'infestation jusqu'aux dégâts, que nous avons entrepris d'élaborer un modèle de diminution de rendement, composé de 6 sous-modèles décrivant la ponte, la durée de développement larvaire (Got, 1985, 1987; Got et Rodolphe, 1989), la mortalité larvaire (Got, 1985), la position des larves sur le plant (Labatte, 1988), la réalisation des dégats (Labatte, 1988) et la perte de rendement provoquée par les galeries. Cet article présente la validation du modèle de durée de développement. 
De nombreux facteurs influencent la durée de développement des stades larvaires de la pyrale (Guennelon et Audemard, 1960; Stengel et Schubert, 1982; Lee et Spence, 1987). Cependant, il n'est pas possible actuellement de prendre en considération tous ces facteurs, car leur action, peu importante pour certains, n'est pas suffisamment connue et leur nombre trop important. Les études bibliographiques démontrent que le principal facteur à prendre en compte est la température (Caffrey et Worthley, 1927; Matteson et Decker, 1965; Sharpe et de Michele, 1977; Beck, 1983; Wagner et al, 1984a; Onstad, 1988). D'autre part, de nombreux auteurs ont souligné la nécessité de prendre en compte la variabilité au sein d'une population (Loewer, 1973; Stinner et al, 1975; Rodolphe et al, 1977, Sharpe et de Michèle, 1977; Sharpe et al, 1977; Barfield et al, 1978; Curry et al, 1978; Wada et Kobayashi, 1980; Brown, 1982; Johnson et al, 1983; Wagner et al, 1984b; Kemp et al, 1986; Hudes et Shoemaker, 1988). Aussi, avons-nous choisi de tester, dans un premier temps, un modèle simple ne décrivant que l'action de la température et la variabilité individuelle du développement au sein d'une population. D'autres facteurs ne seront intégrés que si leur influence altère la validité du modèle.

La variabilité de la durée de développement au sein d'une population est classiquement représentée par une fonction de distribution. Le choix d'une loi n'est alors déterminé que par la forme des données. Des distributions normales ont été choisies pour représenter cette variabilité, compte tenu de leur bonne adéquation aux données et de leur simplicité : elles facilitent en effet les calculs (Got et Rodolphe, 1989), et ont des paramètres (moyennes et écarts types) qui sont aisément interprétables.

De nombreux modèles ont été proposés pour décrire la relation entre vitesse de développement et température. Ces modèles sont étudiés et comparés dans la synthèse bibliographique de Wagner et al (1984a). Le modèle considéré comme optimal est estimé à partir de données de laboratoire. Pour la pyrale, les données de laboratoire existant dans la littérature correspondent à des expériences en températures constantes, notamment pour les températures extrêmes, et à des conditions d'alimentation (feuilles et morceaux de cannes de maïs coupés, haricot vert) très différentes des conditions naturelles (Caffrey et Worthley, 1927; Matteson et Decker, 1965). La validation d'un modèle estimé à partir de ces données reviendrait donc à tester simultanément les hypothèses du modèle et la validité des données de laboratoire, sans pouvoir identifier la part d'erreur qui leur est respectivement due. D'autre part, l'estimation en plein champ de modèles complexes, comparativement aux sommes de degrés-jours, implique des expériences et des calculs lourds qui ne peuvent se justifier indépendamment de la précision à atteindre.

Compte tenu des connaissances déjà acquises sur la pyrale, nous avons choisi de travailler dans un premier temps sur le modèle le plus simple, le modèle de degrés-jours, qui suppose une relation linéaire entre vitesse de développement et température. En effet, les températures moyennes journalières estivales en France correspondent systématiquement à la zone de linéarité décrite par les différents auteurs ayant travaillé sur le développement larvaire de la pyrale en laboratoire. Caffrey et Worthley (1927) estiment, à partir d'expérimentations en températures constantes que la vitesse de développement peut être considérée comme une fonction linéaire de la température entre 16 et $27^{\circ} \mathrm{C}$, la vitesse maximale de développement se situant à $34{ }^{\circ} \mathrm{C}$; les expériences de Matteson et Decker (1965) mettent en évidence une relation linéaire entre 15 et $32{ }^{\circ} \mathrm{C}$, à partir d'expérimentations en températures constantes ou de thermopériodes de faibles amplitudes.

Ainsi, le modèle étudié (Got et Rodolphe, 1989) est composé de 2 fonctions : un modèle de degrés-jours qui permet de calculer l'âge physiologique d'une cohorte de larves, et 4 lois normales qui représentent la variabilité du développement dans la population pour les 4 premiers stades larvaires (fig 1).

Ce modèle a été estimé, testé et validé en région parisienne, sur 2 variétés, LG11 et DEA, à partir de 11 suivis du développement larvaire ( 9 sur LG11 et 2 sur DEA) correspondant à des séquences climatiques et phénologiques du maïs différentes (Got, 1987, Got et Rodd, 1989; Lacan, 1989; Smits, 1989; Stephan, 1989). Ces premiers résultats ont montré que le modèle représentait correctement le développement des différentes populations suivies dans les conditions de l'expérience.

Cet article présente la validation en plein champ du modèle, dont les paramètres ont été estimés en 1983, 1984 et 1986 . Cette validation a été réalisée sur la variété DEA dans 4 sites géographiques, Avignon, Bordeaux, Colmar, Versailles, et sur les variétés LG11 et DEA (Versailles, 1988) et ISORA (Avignon, 1989). Nous 
a

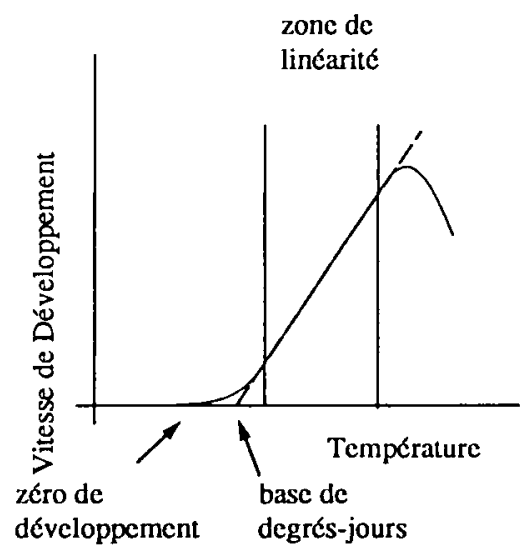

b Loi normale

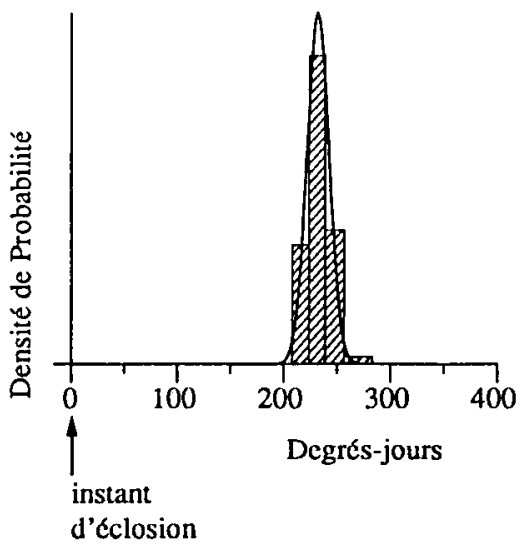

Fig 1. Modèle de durée de développement. a. Le modèle de degrés-jours (- - ) repose sur l'hypothèse que la relation entre vitesse de développement et température $(-)$ peut être considérée comme linéaire dans une certaine gamme de températures. L'âge physiologique moyen d'une cohorte de larves à un instant donné peut être calculé dans ce cas comme la somme des températures supérieures à la base de degrés-jours $\left(10^{\circ} \mathrm{C}\right.$ pour la pyrale), depuis l'entrée dans le stade jusqu'à l'instant considéré. b. Quatre lois normales décrivent la variabilité de la durée des stades au sein d'une population. La figure représente l'ajustement d'une loi normale sur des données de laboratoire (Meusnier, 1986, données non publiées). L'histogramme figure le nombre de larves entrées dans le $5^{\mathrm{e}}$ stade entre les différents instants d'observation. La courbe continue figure la loi normale ajustée.

nous sommes plus particulièrement attachés à évaluer le réalisme du modèle de degrés-jours, qui détermine l'échelle de temps physiologique choisie, afin de préciser son adéquation aux différents objectifs pour lesquels le modèle de développement peut être utilisé, prévisions agronomiques ou études physiologiques plus fondamentales.

\section{MATÉRIEL ET MÉTHODES}

Dans chaque site, le développement a été suivi à partir d'infestations artificielles, dont les principales ca- ractéristiques sont récapitulées dans le tableau 1 . L'utilisation de pontes de laboratoire présente 2 avantages: les caractéristiques de la population sont connues; les infestations artificielles permettent de choisir les dates d'infestation, et donc de tester l'influence d'alimentations liées au stade phénologique du maïs et d'étudier des séquences climatiques différentes au cours d'une même année. Il sera cependant nécessaire de valider les résultats obtenus sur des infestations naturelles. Les expérimentations ont été réalisées avec la variété de maïs DEA, qui est la plus répandue en France actuellement (ONIC, 1988). La variété DEA n'étant pas du tout cultivée dans le SudEst de la France, une variété supplémentaire, locale, ISORA, a été suivie à Avignon. Les dates d'infestation ont été choisies afin de représenter le début et la fin

Tableau I. Validation 1989 : taux d'infestation et stade phénologique du maïs au moment du dépôt des œufs pour les différents sites et dates d'infestation.

\begin{tabular}{lcccccccc}
\hline $\begin{array}{c}\text { Site } \\
\text { Caractéristiques }\end{array}$ & Avignon & Bordeaux & & Colmar & Versailles \\
\hline $\begin{array}{l}\text { Date d'infestation } \\
\begin{array}{l}\text { Taux } \\
\text { (ooplaque/plante) }\end{array}\end{array}$ & 14 juin & 25 juillet & 19 juin & 11 juillet & 7 juillet & 25 juillet & 10 juillet & 24 juillet \\
$\begin{array}{l}\text { Stade phénologique } \\
\text { du maïs }\end{array}$ & Cornet & Post-Flor & PS & Post-Flor & PS & Post-Flor & PS & Floraison \\
\end{tabular}

PS : Panicule sensible; Post-Flor : postérieur à la fin de la floraison femelle. 
des infestations naturelles habituelles de la région pour Bordeaux, Colmar et Versailles. À Avignon, les populations de pyrales étant bivoltines, les dates choisies correspondent au début de chaque génération habituellement observé (Guennelon et Audemard, 1960). Le taux d'infestation était d'une ponte par plante dans tous les sites sauf à Versailles, où ce taux était de 2 pontes par plante afin de pouvoir estimer et tester à partir des mêmes expérimentations le modèle de comportement larvaire.

Chaque infestation a été réalisée à partir d'une cohorte d'œufs pondus en une nuit, produite par le laboratoire du Magneraud (INRA). Les œufs, reposant sur un papier filtre, ont été épinglés sur la face inférieure des feuilles au stade "tête noire", de manière à limiter les risques de dessèchement des pontes. Leur incubation a été suivie sur 100 plants bordant les parcelles de prélèvements (fig 2), afin de déterminer la courbe d'éclosion, point initial du développement larvaire.

Le protocole d'expérimentation a été mis au point à partir des expérimentations de 1988 réalisées à Versailles (Lacan, 1989; Smits, 1989; Stephan, 1989). Un échantillonnage allégé a été déterminé pour être réalisable par une seule personne. Le critère de sélection de l'échantillonnage a été la possibilité d'obtenir des estimations comparables à celles obtenues avec l'échantillonnage complet. Un tel échantillonnage permet donc a fortiori une validation précise du modèle déjà estimé. Ces traitements ont montré que 4 dissections de 10 plants lors des changements de chacun des stades permettraient une estimation précise du modèle. La période de changement de chaque stade $(\mu \pm 2 \sigma)$ a été déterminée à partir des paramètres du modèle (tableau il). Compte tenu du recouvrement des stades, 11 sommes de températures ont été ainsi fixées, auxquelles les prélèvements ont été approximativement réalisés : $20,40,60,80,110,130,160$, $210,260,310,360$ degrés-jours. Afin d'en déterminer la date, un calcul quotidien de la somme des températures était réalisé à partir de $50 \%$ d'éclosion.

Ce protocole suppose que la mortalité est comparable d'une expérimentation à l'autre, ce qui est peu probable. Aussi, les effectifs minimaux de larves par prélèvement permettant de garantir la qualité de la validation ont été déterminés. Des tests comparables aux précédents ont montré que 50 larves par prélèvements étaient suffisantes pour estimer les durées des stades 1 et 2 , et 20 larves par prélèvement pour les 3 derniers stades. En cas de mortalité importante, les

\begin{tabular}{|c|c|c|c|c|c|c|}
\hline \multicolumn{7}{|c|}{ Suivi des pontes naturelles (50 plants) } \\
\hline \multicolumn{4}{|c|}{ Survi de leclosion ( 50 plants) } & & & \\
\hline 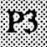 & I.6. & $\mathrm{pq}$ & P12. & P. & 1818 & \\
\hline 12 & P5: & 28 & P11 & P14 & PI & \\
\hline PI. & $\mathrm{P4}$ & $\mathrm{P} 7$ & $P 10$ & P13 & 916 & \\
\hline
\end{tabular}

Suivi des pontes naturelles ( 50 plants)

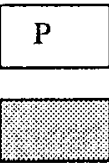

Prélèvement de 10 plants

Plants infestés

Fig 2. Plan d'expérience de la validation de 1989 pour une variété et une date d'infestation.

prélèvements ont été augmentés, dans la limite des plantes disponibles. Un protocole entièrement fonction du nombre de larves n'a pas été choisi en raison des difficultés de mise en place et de planification qu'il comportait.

Nous avons considéré que la population issue d'une infestation artificielle est répartie spatialement de façon régulière, hypothèse confortée par la cohérence des ajustements déjà réalisés (Got, 1987; Got et Rodolphe, 1989). Nous n'avons donc pas effectué de tirages aléatoires des plants. Les prélèvements étaient disposés sur 3 rangs contigus. Les dissections ont été réalisées progressivement sur les 3 rangs afin de réduire le temps pendant lequel des plants étaient en bordure de la clairière de dissection (fig 2). Les plants attenant aux prélèvements ont été également infestés, afin de garantir l'homogénéité du taux d'infestation.

La détermination des stades est faite à partir d'une méthode de discrimination, basée sur un modèle de répartition des tailles de capsules céphaliques dans les différents stades (Got, 1988).

Dans chaque site, les pontes naturelles ont été suivies sur les parcelles d'expérimentation afin d'évaluer les risques de biais dus à des infestations "parasites".

Tableau II. Paramètres estimés du modèle en 1983, 1984 et 1986 : moyennes $(\mu)$ et écarts types $(\sigma)$ de la durée des stades et des stades cumulés, en degrés-jours (base 10).

\begin{tabular}{crrrrrrr}
\hline $\begin{array}{c}\text { Paramètres } \\
\text { estimés }\end{array}$ & $L_{1}$ & $L_{2}$ & $L_{3}$ & $L_{4}$ & $L_{1}+L_{2}$ & $L_{1}+L_{2}+L_{3}$ & $L_{1}+L_{2}+L_{3}+L_{4}$ \\
\hline$\mu$ & 67 & 34 & 63 & 84 & 101 & 165 & 249 \\
$\sigma$ & 16 & 9 & 31 & 32 & 18 & 36 & 48 \\
\hline
\end{tabular}


Des enregistrements météorologiques étaient disponibles dans chaque site. À Avignon et à Bordeaux, les enregistrements sont tri-horaires (matériel Cimel, Stefce, Bioclimatologie, INRA); à Colmar, les enregistrements sont horaires (Météorologie nationale); à Versailles, les enregistrements sont horaires (matériel Campbell Scientific Ltd, Zoologie). Les degrés-jours ont été calculés comme la somme horaire ou trihoraire des températures moyennes au dessus de $10^{\circ} \mathrm{C}(D=\Sigma$ (température-10)/24 pour les moyennes horaires, $D=\Sigma$ (température-10)/8 pour les moyennes tri-horaires, pour les températures supérieures à la base). Les conditions de transport n'étant pas connues, le cumul de degrés-jours a débuté à partir de $50 \%$ d'éclosion, premier repère chronologique connu qui ait une signification biologique.

\section{RÉSULTATS}

Le traitement comporte 3 étapes : l'estimation de la courbe d'éclosion, la validation du modèle et l'estimation du modèle de durée de développement sur les données de l'expérience.

\section{Le modèle d'éclosion choisi}

C'est le même que celui représentant la durée de développement des stades larvaires, compte tenu de la similarité des phénomènes biologiques (loi normale et échelle de degrés-jours). Le seul objectif de ce traitement est de disposer, en plein champ, d'un point initial de démarrage du développement larvaire comparable d'une infestation à l'autre. La moyenne de la courbe d'éclosion permet de calculer une échelle de degrés-jours débutant à $50 \%$ de l'éclosion, ne prenant pas en compte la période d'incubation sur le terrain. La soustraction de la variance de l'éclosion à la variance de la durée des stades permet d'étudier sans biais expérimental la variabilité des stades. Cette étape est uniquement technique et ne présente pas d'intérêt biologique particulier. Elle n'est pas développée ici.

\section{La validation du modèle de développement}

Elle consiste à comparer graphiquement les prévisions du modèle et les observations. L'écart entre le modèle et les données permet d'évaluer la qualité prédictive du modèle.

\section{L'estimation des paramètres du modèle de développement sur les données}

Elle permet d'évaluer la durée moyenne et l'écart type de chaque stade au cours de l'expérience. Nous avons utilisé la durée moyenne estimée pour quantifier la différence entre le modèle et les données en comparant moyennes de l'expérience et moyennes du modèle à valider. Cette différence permet d'évaluer quantitativement la précision des prévisions du modèle et donc son réalisme. Les paramètres du modèle sont estimés par maximum de vraisemblance (Got et Rodolphe, 1989), avec les programmes e04jaf et e04jbf de la bibliothèque de sousprogrammes NAG (1983). L'écart entre modèle et données est évalué en degrés-jours. Sa correspondance en jours est estimée à partir de la température moyenne pendant l'expérimentation.

La figure 3 et le tableau III résument les résultats de la validation.

À Colmar, I'hétérogénéité des œufs utilisés pour la $2^{\mathrm{e}}$ infestation ne garantit pas l'estimation de l'éclosion. Aussi, la durée du premier stade a été estimée. Seule la validation des stades 2, 3 et 4 a pu être réalisée pour cette infestation.

À Avignon, une légère infestation naturelle était présente (4 pontes pour 100 plantes pendant la période de la première infestation, 5 pontes pour 100 plantes pour la $2^{e}$ ). Cette infestation explique une partie de la variabilité des observations.

La mortalité a été importante dans tous les sites $(80-90 \%$ entre l'éclosion et la fin des expérimentations).

À Bordeaux, Colmar et Versailles, les résultats sont très satisfaisants : l'entrée dans le stade $L_{4}$, qui correspond au début de la réalisation de galeries importantes (Labatte, 1988), est prévue à moins de $3 \mathrm{j}$ près. L'entrée dans le dernier stade est prévue à $4 \mathrm{j}$ près. La précision de ces prévisions est donc très bonne pour des objectifs comme la modélisation des pertes de rendement ou la lutte contre les stades larvaires du ravageur. À Avignon, les résultats, tout en étant raisonnables, montrent un décalage plus important (1 semaine pour l'entrée dans le dernier stade).

Cinq phénomènes et facteurs peuvent avoir contribué aux écarts observés : 
a

L1

\section{VALIDATIONS 1989}

\section{VERSAILLES}

L2

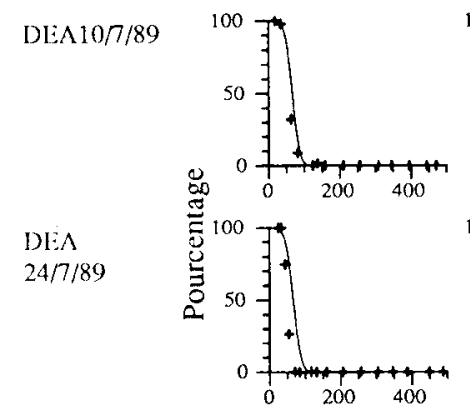

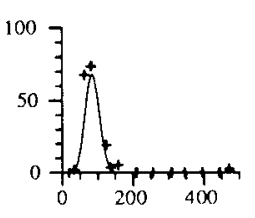

L3
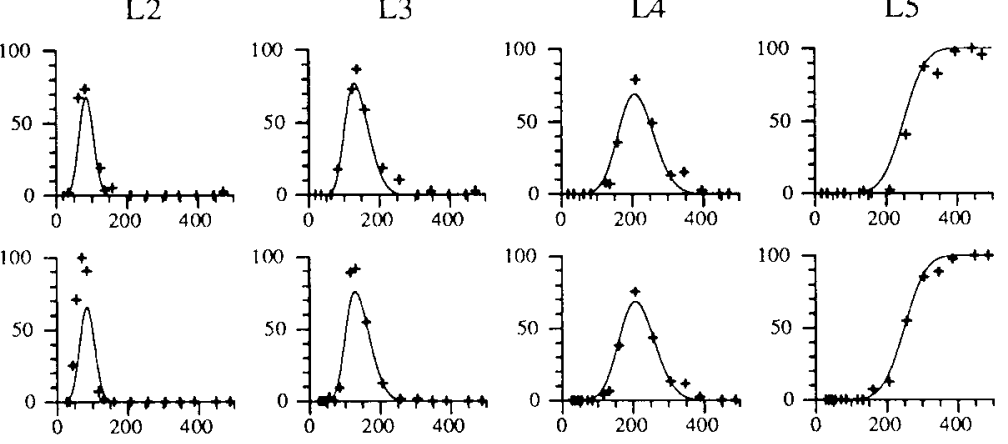

\section{COLMAR}
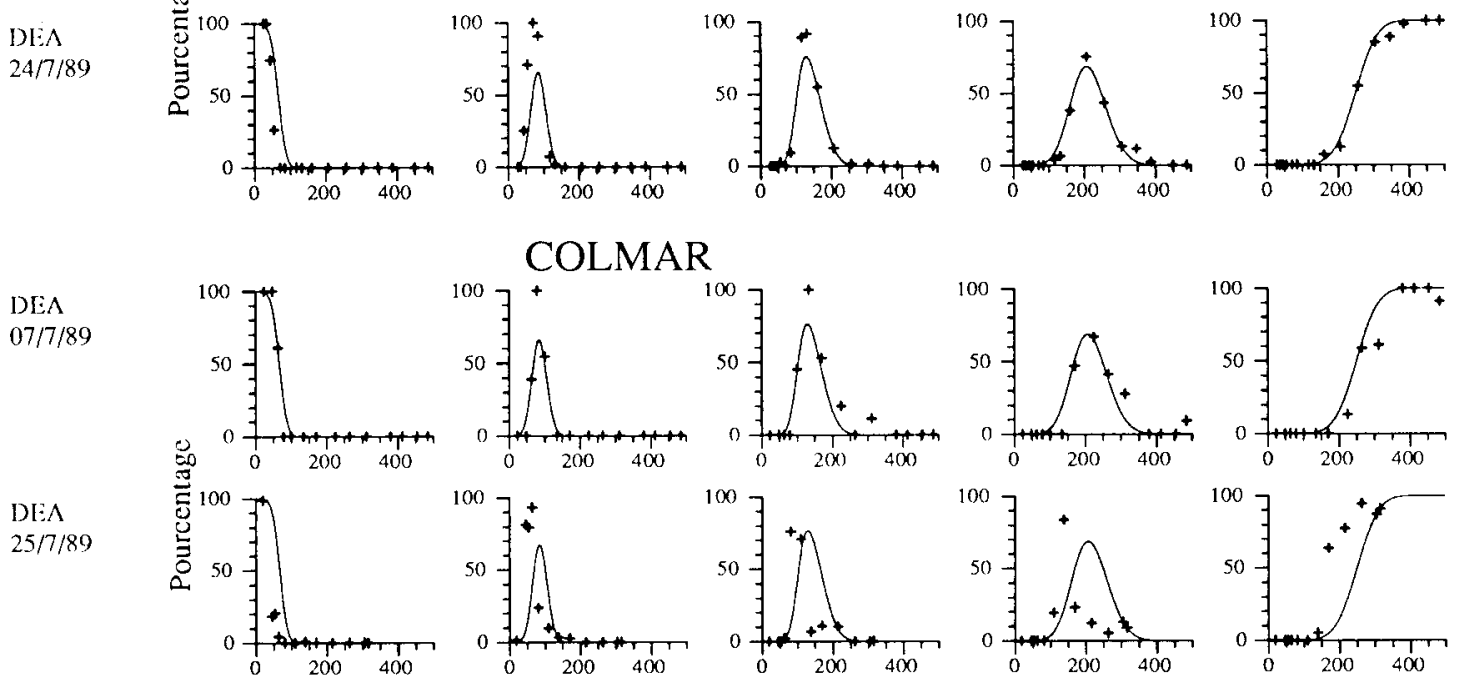

\section{BORDEAUX}
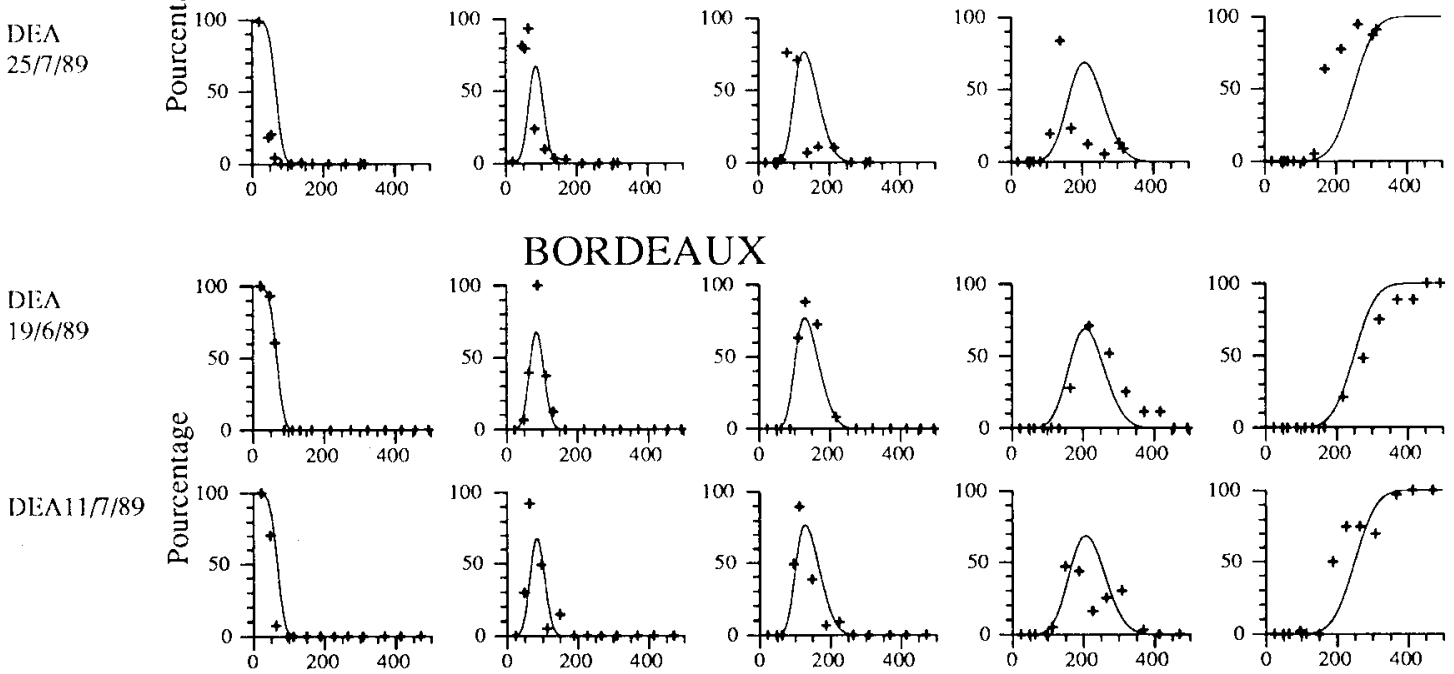

\section{AVIGNON}
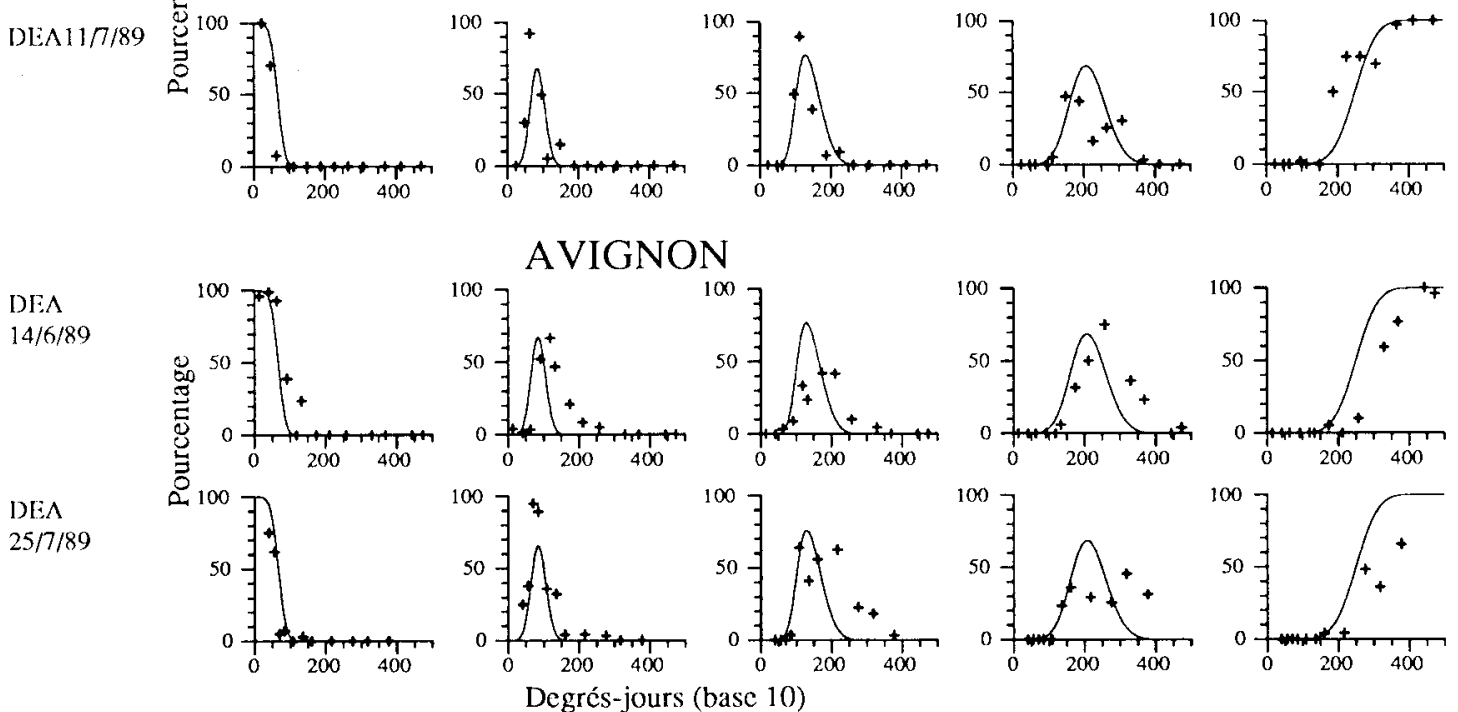

Fig 3. Validation 1989 dans 4 sites géographiques, sur DEA et pour 2 dates d'infestation par site. La qualité du modèle s'évalue par sentées en pourcentage par stade du nombre de larves trouvées à chaque prélèvement (a) ou en nombre de larves (b) en fonction dérant les effectifs de larves dans chaque stade pour un prélèvement comme la réalisation d'une multinomiale $\left(N_{i} p_{i l}\left(1-p_{i l}\right)\right.$, où $i$ est prélèvement $i$ ). 
LI

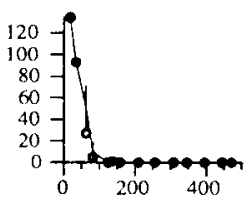

DEA

$24 / 7 / 89$

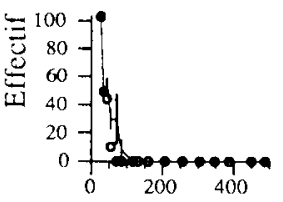

DEA

$07 / 7 / 89$

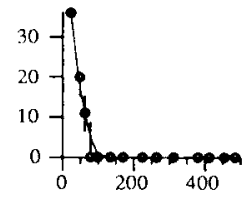

DEA

$25 / 7 / 89$

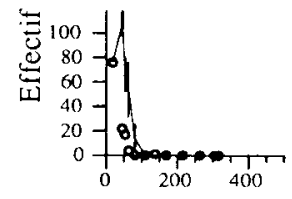

DISA

$19 / 6 / 89$

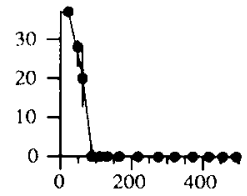

DI:A

$11 / 7 / 89$
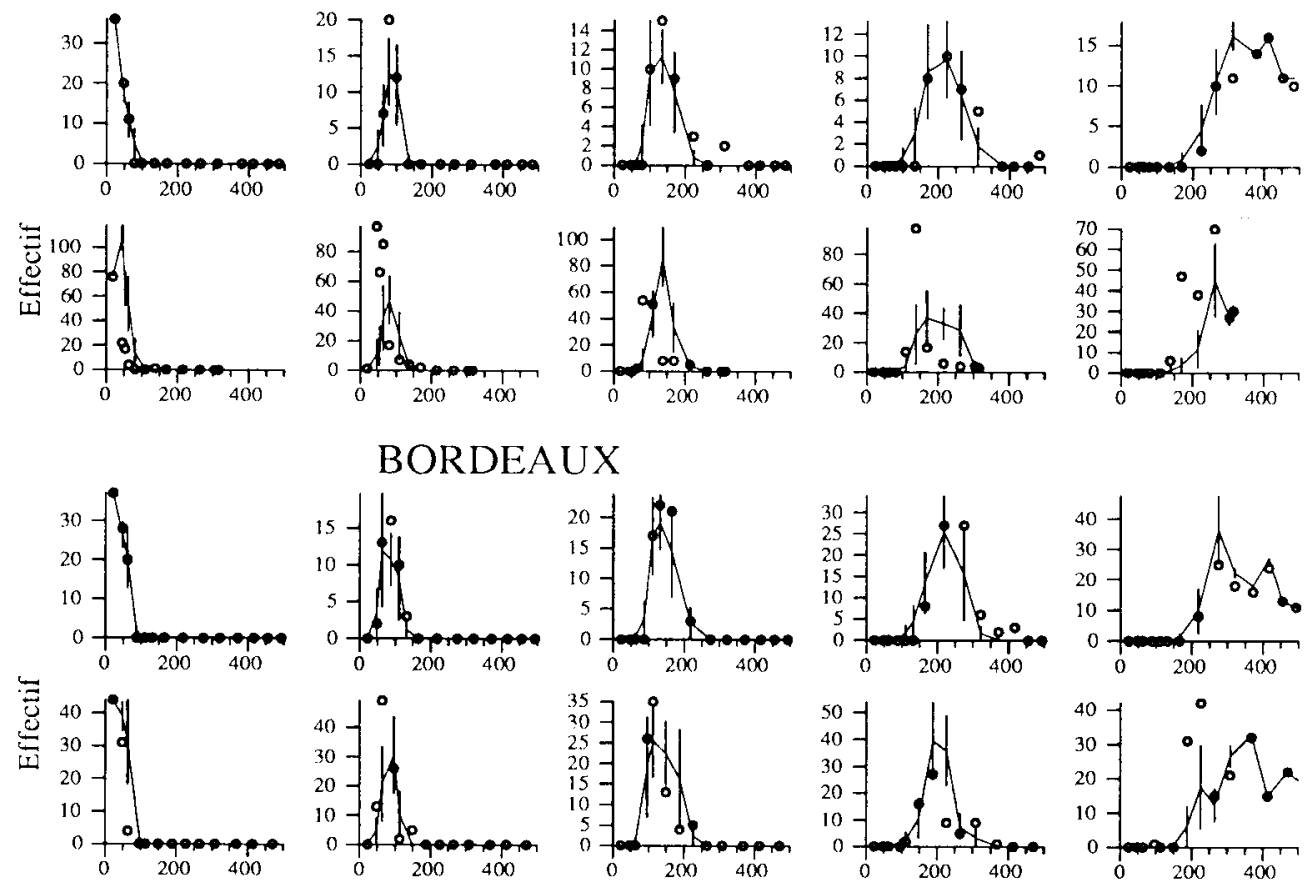

BORDEAUX
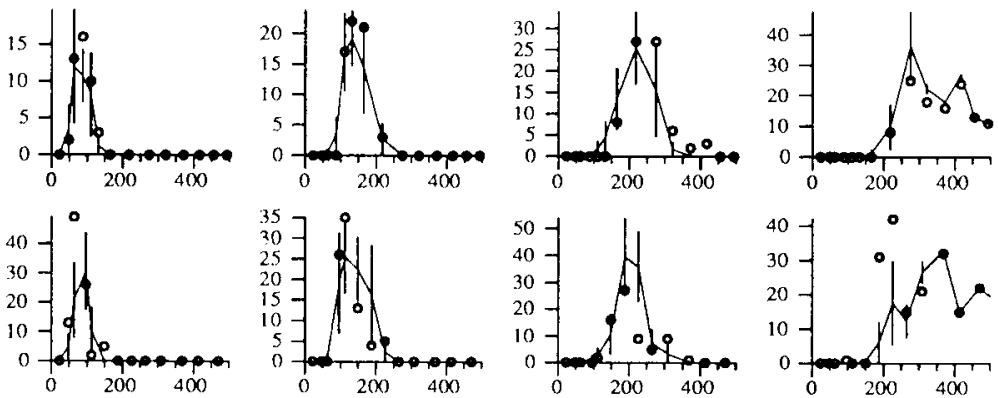

AVIGNON
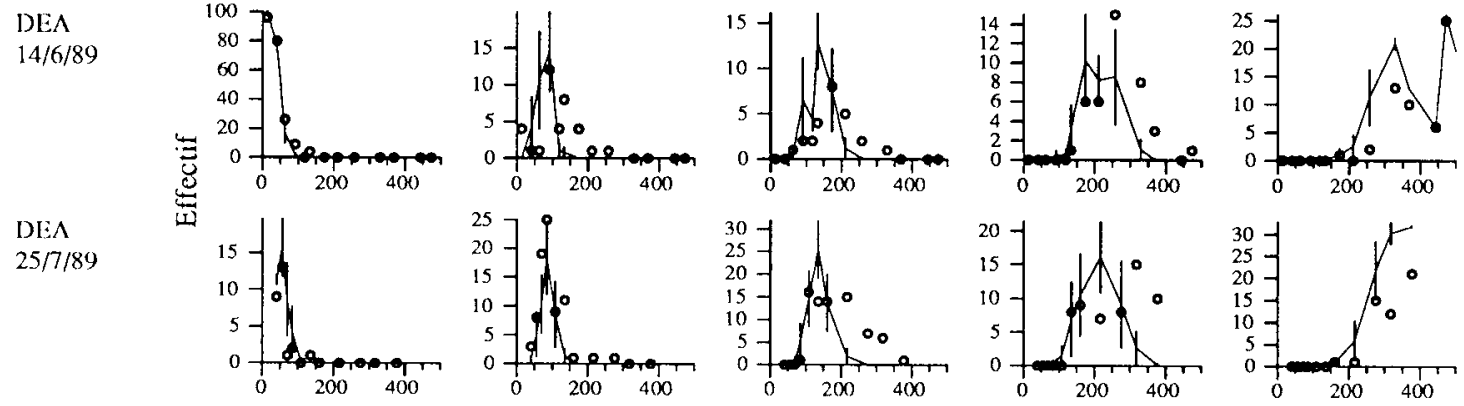

Degrés-jours (base 10)

l'écart entre les prévisions du modèle (courbe continue) et les données observées (points). Prévisions et observations sont repréd'une échelle de degrés-jours en base 10. Les traits verticaux (b) représentent les écarts types des observations, calculés en consile prélèvement, / le stade larvaire, $p_{i l}$ la probabilité d'avoir une larve dans le stade $I$, et $N_{j}$ le nombre total de larves trouvées pour le 
Tableau III. Validation du modèle; différences entre moyennes d'entrée dans les 3 derniers stades estimées sur les données de 1989 et paramètres du modèle estimés en 1983, 1984 et 1986 .

\begin{tabular}{|c|c|c|c|c|c|c|c|}
\hline \multirow[t]{2}{*}{ Infestation } & \multicolumn{2}{|c|}{ Entrée Stade $L_{3}$} & \multicolumn{2}{|c|}{ Entrée Stade $L_{4}$} & \multicolumn{2}{|c|}{ Entrée Stade $L_{5}$} & \multirow{2}{*}{$\begin{array}{l}\text { Température Moyenne } \\
\text { (période expérimentale) }\end{array}$} \\
\hline & Degrés-jours & Jours & Degrés-jours & Jours & Degrés-jours & Jours & \\
\hline \multicolumn{8}{|l|}{ Versailles } \\
\hline DEA 10 juillet & +3 & +0 & +30 & +3 & +40 & +4 & $18,9^{\circ} \mathrm{C}$ \\
\hline DEA 24 juillet & -1 & -0 & +10 & +1 & +10 & +1 & \\
\hline \multicolumn{8}{|l|}{ Avignon } \\
\hline DEA 15 juin & +52 & +4 & +43 & +3 & +70 & +5 & $23,2^{\circ} \mathrm{C}$ \\
\hline DEA 25 juillet & +22 & +2 & +60 & +5 & +80 & +6 & \\
\hline ISO 15 juin & +52 & +4 & +75 & +6 & +90 & +7 & \\
\hline ISO 25 juillet & +10 & +1 & +30 & +2 & +70 & +5 & \\
\hline \multicolumn{8}{|l|}{ Bordeaux } \\
\hline DEA 19 juin & +9 & +1 & +15 & +1 & +35 & +3 & $21,5^{\circ} \mathrm{C}$ \\
\hline DEA 11 juillet & -3 & -0 & -12 & -1 & -35 & -3 & \\
\hline \multicolumn{8}{|l|}{ Colmar } \\
\hline DEA 7 juillet & -1 & -0 & +30 & +3 & +30 & +3 & $19,0^{\circ} \mathrm{C}$ \\
\hline DEA 25 juillet & - & - & -10 & -1 & -30 & -3 & \\
\hline
\end{tabular}

\section{La variabilité des échantillonnages}

Elle a été beaucoup plus importante lors de la deuxième infestation, à Avignon, à Bordeaux et Colmar, ce qui se traduit par une croissance et une décroissance irrégulière des effectifs par stade. L'origine de cette hétérogénéité n'a pas été identifiée. Elle peut être due aux conditions d'incubation des œufs, aux conditions climatiques ou culturales. Dans les sites où cette variabilité est importante, le modèle et, par suite, l'écart entre modèle et données, sont estimés moins précisément. II faut cependant noter que le nombre de points par stade et la cohérence des apparitions et disparitions d'un stade sont suffisants pour la validation. Les échantillonnages de Versailles, qui correspondent au site de détermination du protocole, sont satisfaisants.

\section{Le taux de diapause}

Estimé comme le taux de larves qui n'a pas nymphosé, il est variable selon les sites et les dates d'infestation. II est important (environ 50\%) pour la première infestation à Avignon et pour les 2 infestations de Bordeaux. II est supérieur à $90 \%$ pour les premières infestations de Colmar et
Versailles. Il est égal à 100\% pour les deuxièmes infestations d'Avignon, Colmar et Versailles. On peut remarquer que la deuxième infestation présente systématiquement un développement plus rapide que celui observé pour la première infestation. Les résultats obtenus et les connaissances actuelles ne permettent cependant pas d'identifier une interaction particulière entre diapause et développement.

\section{L'alimentation}

L'alimentation des larves des 2 infestations d'un même site a été différente. En effet, les larves de la seconde infestation étaient majoritairement situées sur l'épi, alors que pour la première infestation, les larves étaient à l'aisselle des feuilles et dans la panicule avant la pollinisation, puis se dispersaient le long du plant après la pollinisation. Malgré la similitude de ce comportement dans les différents sites, la différence de développement entre les 2 dates d'infestation, quantifiée à partir de l'entrée moyenne dans le dernier stade, est très variable selon le site : 20 degrésjours à Avignon, 30 degrés-jours à Versailles, 60 degrés-jours à Colmar et 70 degrés-jours à Bordeaux. On ne peut donc espérer dégager une relation simple de l'action de l'alimentation. 


\section{La variété et le stade phénologique du maïs}

Ces deux paramètres qui conditionnent aussi l'alimentation, ne semblent intervenir que faiblement dans les expériences réalisées. Les paramètres du modèle ont été estimés sur la variété LG11. Les expérimentations de 1988 réalisées simultanément sur LG11 et DEA, à Versailles (fig 4 et tableau IV), les résultats de la validation sur DEA à Versailles en 1989 (fig 3) et les résultats de la validation 1989 à Avignon sur ISORA (fig 5, tableau III), montrent que les écarts entre DEA, LG11 et ISORA sont très réduits ( $3 j$ de différence au plus entre 2 variétés). Les variétés ISORA et DEA sont au stade cornet légèrement moins favorable au développement de la pyrale.

\section{La température}

C'est le facteur qui a présenté la plus grande variabilité d'un site à l'autre (fig 6). Les valeurs élevées observées à Avignon et à Bordeaux peuvent altérer le fonctionnement du modèle. En effet, si les moyennes journalières correspondent systématiquement à la zone de linéarité du modèle de degrés-jours, il n'en est pas de même des moyennes tri-horaires, horaires et des maxima journaliers. Les premiers tests réalisés pour tester cette hypothèse n'ont cependant pas été concluants.

Pour évaluer l'impact d'une surestimation du développement aux températures élevées, la somme de températures cumulées à Avignon a

Tableau IV. Validation du modèle; différences entre moyennes d'entrée dans les 3 derniers stades estimées sur les données de 1988 et paramètres du modèle estimés en 1983, 1984 et 1986.

\begin{tabular}{|c|c|c|c|c|c|c|c|}
\hline \multirow{2}{*}{$\begin{array}{l}\text { Infestation } \\
\text { Versailles }\end{array}$} & \multicolumn{2}{|c|}{ Entrée Stade $L_{3}$} & \multicolumn{2}{|c|}{ Entrée Stade $L_{4}$} & \multicolumn{2}{|c|}{ Entrée Stade $L_{5}$} & \multirow{2}{*}{$\begin{array}{c}\text { Température Moyenne } \\
\text { (juillet-août) }\end{array}$} \\
\hline & Degrés-jours & Jours & Degrés-jours & Jours & Degrés-jours & Jours & \\
\hline DEA 13 juillet & +3 & +0 & +18 & +3 & +30 & +4 & $17,2^{\circ} \mathrm{C}$ \\
\hline DEA 26 juillet & -1 & -0 & +1 & +0 & 0 & 0 & $17,2^{\circ} \mathrm{C}$ \\
\hline LG11 13 juillet & +5 & +1 & -1 & -0 & +9 & +1 & $17,2^{\circ} \mathrm{C}$ \\
\hline LG11 26 juillet & -12 & -2 & -6 & -1 & -7 & -1 & $17,2^{\circ} \mathrm{C}$ \\
\hline
\end{tabular}

L1

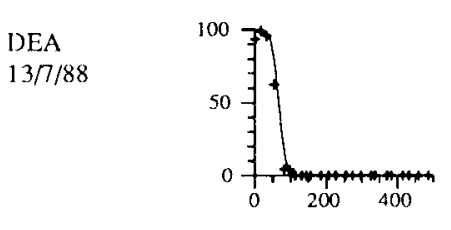

DEA
$26 \pi / 88$

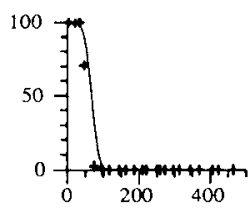

LG1 1

$137 / 88$

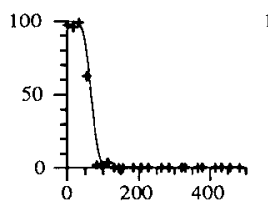

LG11

26/7/88

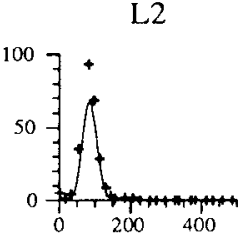

L3
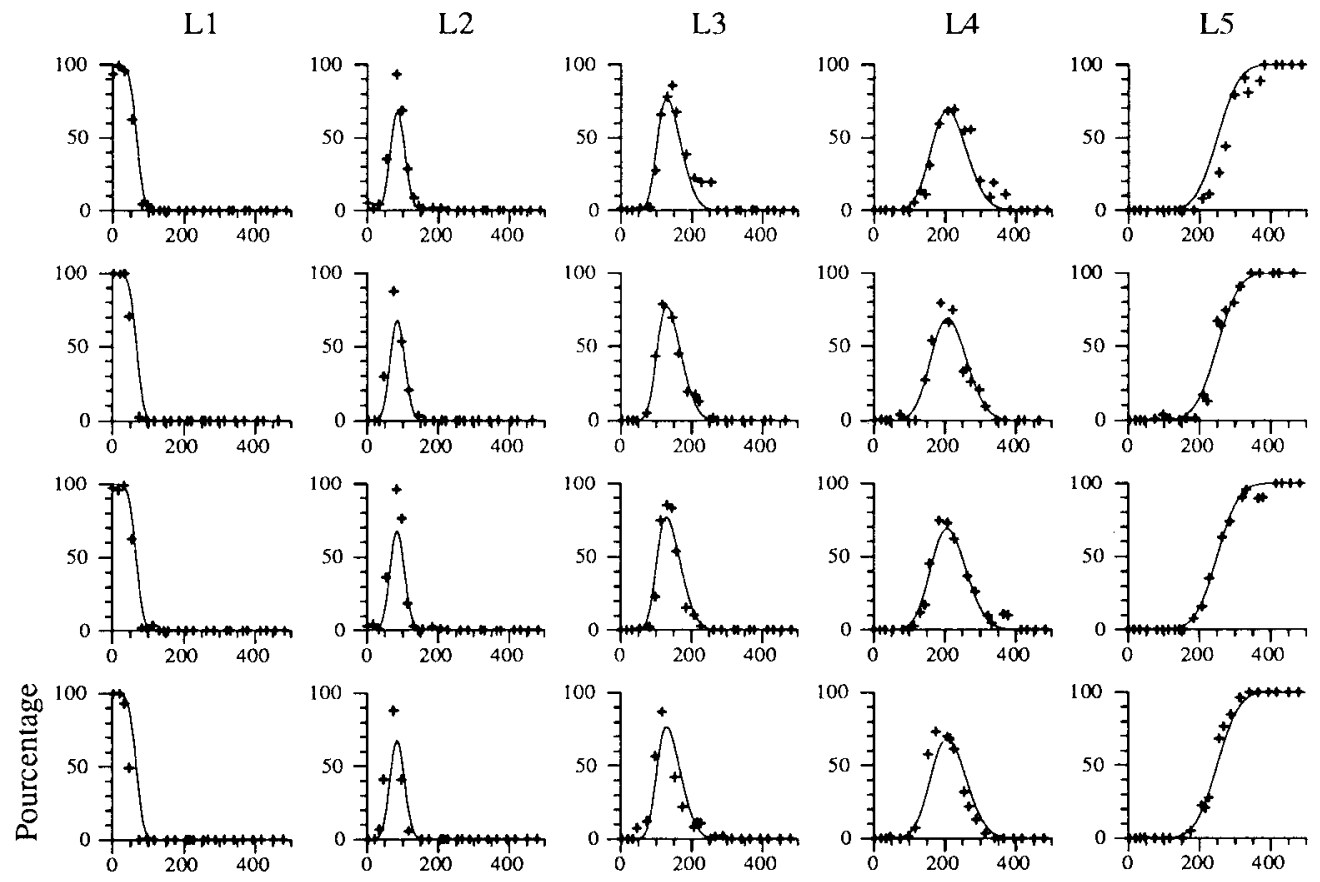

Fig 4. Validation 1988 sur 2 variétés de maïs et pour 2 dates d'infestation, en région parisienne. La qualité du modèle s'évalue par l'écart entre les prévisions du modèle (courbe continue) et les données observées (points). Prévisions et observations sont représentées en pourcentage, en fonction d'une échelle de degrés-jours en base 10. 
L1 L2

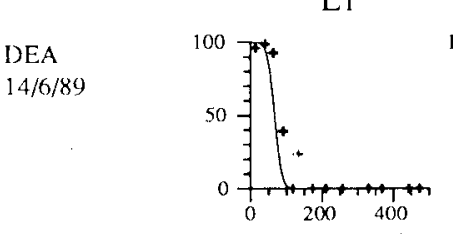

ISORA

$14 / 6 / 89$
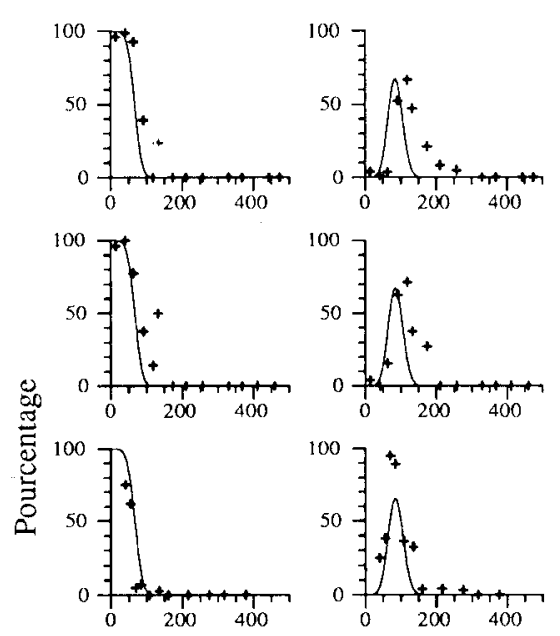

DEA

$25 / 7 / 89$

ISORA
$25 / 7 / 89$
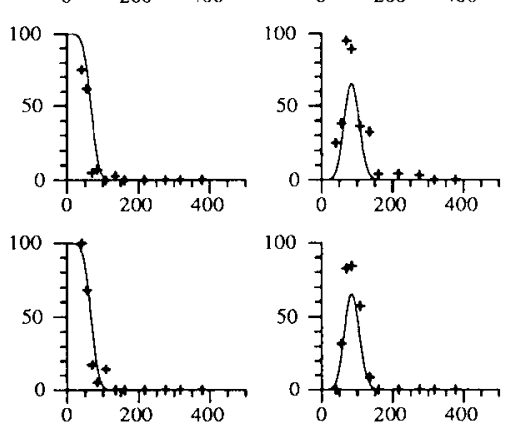

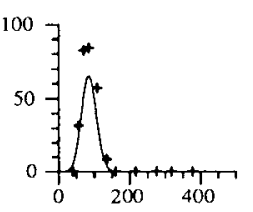

Degrés-jours (base 10)
L3
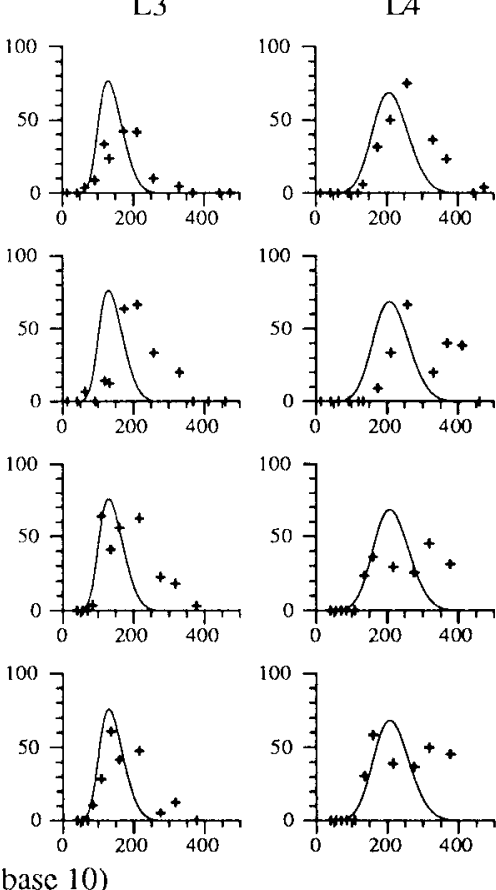

L5
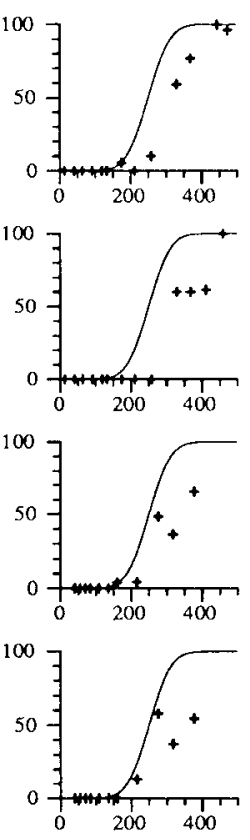

Fig 5. Validation 1989 à Avignon. Comparaison des variétés DEA el ISORA pour les 2 dates d'infestation. Les prévisions du modèle sont figurées par les courbes continues et les données observées par les points.

été calculée en écrêtant les températures audelà de $30^{\circ} \mathrm{C}$. Afin de prendre en compte l'incidence du rythme des mesures climatiques, cet écrêtage a été réalisé à partir de 3 enregistrements de températures différents : la température moyenne tri-horaire, les minima et maxima journaliers à partir desquels l'évolution de la température journalière est simulée à l'aide d'une sinusoïde (Allen, 1971), les enregistrements instantanés enregistrés sur thermographe. Les écrêtages ainsi réalisés ne permettent de diminuer l'écart du modèle aux données que d'une dizaine de degrés-jours dans le meilleur des cas (environ 1 jour).

D'autre part, il faut noter que pendant certaines périodes, la température enregistrée à Bordeaux était supérieure à celle d'Avignon (période du 15-21 juillet). L'utilisation d'un modèle qui écrête de manière plus importante la somme de températures, soit par une relation non linéaire, soit par le choix d'un seuil d'écrêtage plus bas, conduirait à améliorer les résultats d'Avignon, en dégradant ceux de Bordeaux, voire ceux de Colmar et Versailles. De plus, les températures enregistrées à $2 \mathrm{~m}$ sous abri étaient presque systématiquement inférieures à la température optimale de développement déterminée par Caffrey et Worthley $(1927)\left(34^{\circ} \mathrm{C}\right)$ et Matteson et Decker $(1965)\left(33^{\circ} \mathrm{C}\right)$. Un mo- dèle non linéaire dont la décroissance commence au-delà des températures enregistrées ne peut donc permettre d'améliorer notablement les prévisions.

\section{DISCUSSION}

Les connaissances sur l'influence de la diapause, de l'alimentation (liée au stade phénologique), et/ou la variété de maïs, ne sont pas suffisantes pour pouvoir quantifier l'action éventuelle de ces facteurs et améliorer les prévisions. Leur prise en compte impliquerait de poser de nombreuses hypothèses qui altéreraient la précision du modèle. Compte tenu de leur faible influence apparente dans ces expérimentations, il n'est pas actuellement envisagé d'intégrer leur action dans le modèle.

Par contre, la prise en compte de la température peut très probablement être améliorée. En effet l'âge physiologique calculé actuellement à partir du modèle de degrés-jours et de la température à $2 \mathrm{~m}$ comporte 2 biais qui ne peuvent être pris en compte indépendamment l'un de l'autre : le modèle de degrés-jours suppose une relation linéaire entre vitesse de développement et température et ne prend donc pas correctement en compte les températures extrêmes; la 
Température 1989

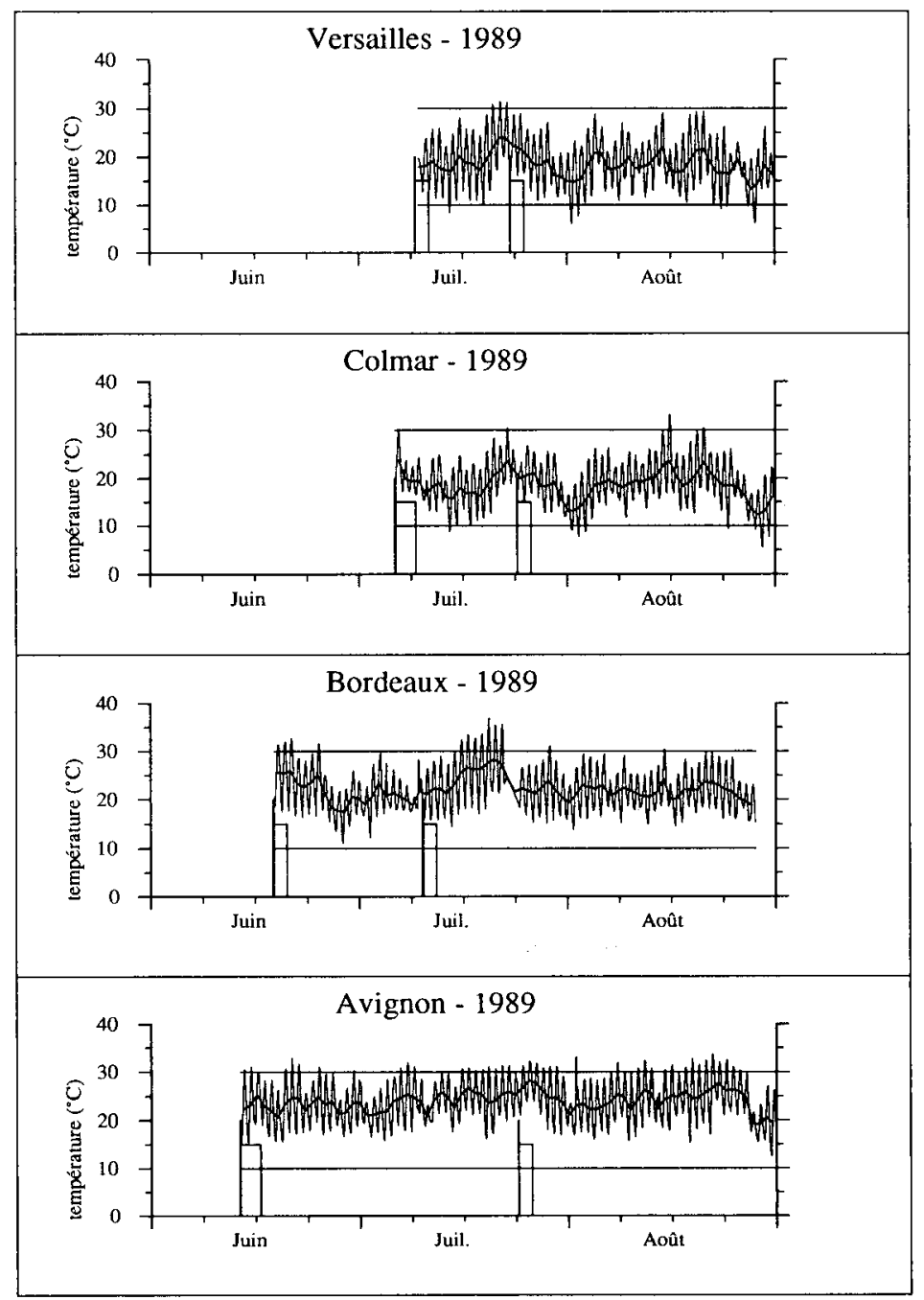

Fig 6. Températures enregistrées en 1989 dans les 4 sites géographiques. Les 2 courbes continues représentent l'évolution des moyennes tri-horaires et journalières de la température à $2 \mathrm{~m}$ sous abri. Les boîtes figurent la période d'éclosion des infestations réalisées. Les seuils critiques de 10 et $30^{\circ} \mathrm{C}$ sont représentés.

température du couvert peut être supérieure à celle mesurée à $2 \mathrm{~m}$ sous abri. Cette dernière hypothèse est confortée par les mesures au sein du couvert que nous avons réalisées au cours de l'expérience en Avignon et à Versailles. En effet, la différence entre température moyenne horaire à $2 \mathrm{~m}$ sous abri et au sein du couvert, dans les tiges, va jusqu'à $12^{\circ} \mathrm{C}$ à Avignon, alors qu'elle est, au maximum, de $4-5^{\circ} \mathrm{C}$ à Versailles. Cette différence est essentiellement conditionnée par le déficit hydrique, le rayonnement et la vitesse du vent (Perrier, 1979). L'étude de ces paramètres dans les 3 sites montre que la situation microclimatique de Bordeaux est beaucoup plus proche de celle de Versailles que de celle d'Avignon. De cette première analyse on peut supposer que les températures subies par les larves sont comparables à celles prises en compte, sauf à Avignon, où elles sont beaucoup plus élevées. Dans ce cas, elles sont alors fréquemment au-delà des températures où la vitesse de développement est maximale, ce qui n'était pas le cas pour les températures enregistrées à $2 \mathrm{~m}$ sous abri. Ceci nous conduit à considérer que la prise en compte de la température du couvert, et d'un modèle non linéaire décrivant la décroissance de la vitesse de développement pour les fortes températures pourrait permettre d'améliorer les simulations réalisées à Avignon sans dégrader celles de Bordeaux.

La validation est une étape indispensable en modélisation. II importe d'interpréter les résultats en fonction des différents objectifs d'utilisation d'un modèle. Pour des objectifs agronomiques, 
la précision obtenue avec le modèle de degrésjours et la température à $2 \mathrm{~m}$ sous abri est satisfaisante. Pour des objectifs où le développement doit être connu plus précisément, la prise en compte de la température du couvert, et d'un modèle non linéaire reliant vitesse de développement et température, doit permettre d'obtenir de meilleurs résultats. Nous allons poursuivre les traitements en ce sens, dans la mesure où ils permettront également d'améliorer la connaissance des relations entre conditions environnementales et précision des prévisions du modèle.

\section{REMERCIEMENTS}

Nous remercions MM Anglade, Galichet, Poitout et Stengel pour avoir accepté d'accueillir et d'encadrer les validations au niveau de leur Station, M Anglade, Croisier, Maginieau et Meynadier pour avoir encadré et assuré la mise en œuvre de l'entretien des cultures dans les différents centres d'expérimentation, $M$ Bleuse de la Météorologie nationale, Mrs Baculat et Valencogne qui nous ont permis d'accéder aux données climatiques des postes météorologiques de chaque centre, MM Schubert et Vible pour leur aide technique.

Nous remercions également MM Leclant, Missonnier, Pierre et Strebler, responsables du DAA "Protection des cultures» de I'INA-PG, et des ENSA de Montpellier et Rennes d'avoir rendu possible la réalisation de ces stages.

Nous remercions enfin $Y$ Durand qui a mis en place les expérimentations dans les différents sites et $S$ Meusnier pour sa participation aux expérimentations et aux traitements.

\section{RÉFÉRENCES}

Allen JC (1976) A modified sine wave method for calculating degree days. Environ Entomo/5, 388-396

Barfield CS, Mitchell ER, Poe SL (1978) A temperature-dependent model of fall armyworm development. Ann Entomol Soc Am 71, 70-74

Beck SD (1983) Thermal and thermoperiodic effects on larval development and diapause in the European Corn Borer, Ostrinia nubilalis. J Insect Physiol 29, 107-112

Brown GC (1982) A generalized phenological forecast model for European Corn Borer. J Kans Entomol Soc 55, 625-638

Caffrey DJ, Worthley LH (1927) A progress report on the investigations of the European corn borer. US Dep Agric Inf Bull 1476, $155 p$

Curry GL, Feldman RM, Sharpe PJH (1978) Foundations of stochastic development. J Theor Biol 74, 397-410
Got B (1985) Approche de la modélisation de la dynamique de population de la pyrale du maîs (Ostrinia nubilalis Hbn Lepidoptera: Pyralidae) dans le Bassin parisien. Thèse de $3^{e}$ cycle, Paris VII, $210 p$

Got B (1987) Une étape dans la construction d'un modèle de dégats de la pyrale du maîs (Ostrinia nubilalis): Le modèle de durée de développement larvaire. CR Séances Acad Agric Fr 73, 179-184

Got B (1988) Determination of Instar of the European Corn borer, Ostrinia nubilalis Hbn. (Lepidoptera: Pyralidae) based on a Distribution Model of Head Capsule Widths. Ann Entomol Soc Am 81, 91-98

Got B, Rodolphe F (1989) Temperature-dependent model for European corn borer (Lepidoptera: Pyralidae) development. Environ Entomol 18, 85-93

Guennelon G, Audemard H (1960) La pyrale du maïs Ostrinia (Pyrausta) nubilitis HBN. (Lépidoptères Pyralidae) dans la basse vallée du Rhône: observations écologiques, incidences économiques. Ann Epiphyt Paris 3, 337-395

Hudes ES, Shoemaker CA (1988) Inferential method for modeling insect phenology and its application to the Spruce Budworm (Lepidoptera: Tortricidae). Environ Entomol 17, 97-108

Johnson DW, Barfield CS, Allen GE (1983) Temperature-dependent developmental model for the velvetbean caterpillar (Lepidoptera: Noctuidae). Environ Entomol 12, 1657-1663

Kemp WP, Dennis B, Beckwith RC (1986) Stochastic phenology model for the Western Spruce Budworm (Lepidoptera: Torticidae). Environ Entomol 15, 547554

Labatte JM (1988) Approche de la modélisation de l'influence des infestations de pyrale (Ostrinia nubilalis $\mathrm{Hbn}$ ) sur le rendement du maïs. Mémoire de DEA, INA-PG, $69 \mathrm{p}$

Lacan GF (1989) Contribution à la validation d'un modèle de développement larvaire de la pyrale du maïs (Ostrinia nubilalis Hübn) dans différentes régions. Mémoire de DAA, spécialité «Protection des cultures», INA-PG, ENSAM, ENSAR, ENSAA, $23 p$

Lee DA, Spence JR (1987) Developmental adaptation of the European corn borer (Ostrinia nubilalis Hübner) in Alberta. Can Entomol 119, 371-380

Loewer OJ (1973) Simulation of the life cycle of the European corn borer. Thesis, Purdue Univ, $194 p$

Lynch RE (1980) European corn borer: yield losses in relation to hybrid and stage of corn development. $J$ Econ Entomol 73, 159-164

Lynch RE, Robinson JF, Berry EC (1980) European corn borer: yield losses and damage resulting from a simulated natural infestation. $J$ Econ Entomol 73, 141-144

Matteson JW, Decker GC (1965) Development of the European Corn Borer at controlled constant and variable temperatures. J Econ Entomol 58, 344-349

Numerical Algorithms Group (1983) Mayfield House, 256 Banbury Road, Oxford OX2 7DE, United Kingdom and 1131 Warren Avenue, Downer's Grove, Iil 60515 , USA, $2600 \mathrm{p}$ 
ONIC (1988) Superficie et répartition variétale, récolte, $28 p$

Onstad D (1988) Simulation model of the population dynamics of Ostrinia nubilalis (Leptidoptera : Pyralidae) in maize. Environ Entomol 17, 969-976

Perrier A (1979) Variation du microclimat d'une culture en fonction de ses caractéristiques biologiques. Bull OEPP 9, 187-204

Rodolphe F, El Shishiny H, Onillon JC (1977) Modélisation de deux populations d'Aleurodes ravageurs des cultures. Congrès de l'AFCET Modélisation et maîtrise des systèmes, 528-535

Sharpe PJH, de Michele DW (1977) Reaction kinetics of poikilotherm development. J Theor Biol 64, 649670

Sharpe PJH, Curry GL, de Michele DW, Cole CL (1977) Distribution model of organism development times. J Theor Biol 66, 21-38

Smits N (1989) Contribution à l'élaboration d'un modèle de dynamique de population d'un ravageur du maïs : validation du modèle de développement larvaire d'Ostrinia nubilalis Hbn (Lepidoptera: Pyralidae). Mémoire de DAA, spécialité «Protection des cultures", INA-PG, ENSAM, ENSAR, ENSAA $19 \mathrm{p}$

Sparks AN, Chiang HC, Triplehorn CA, Guthrie WD (1967) Some factors influencing populations of the European corn borer, Ostrinia nubilalis (Hübner) in the North Central States: Resistance of corn, time of planting and weather conditions Part II, 19581962. lowa Agric Home Econ Exp Stn Res Bull 559, $103 \mathrm{p}$

Stengel M (1969) Influence de l'attaque de la pyrale (Ostrinia nubilalis Hübn Lepidopt Pyralidae) sur le poids et la qualité des grains de Maïs en fonction du type de dégats dans des cultures en Alsace (France). Rev Zool Agric Pathol Veg 10-12, 101107

Stengel M, Schubert G (1982) Etude comparative de la vitesse de croissance et de la sensibilité à la photopériode de deux races de pyrale du maïs (Ostrinia nubilalis Hübn Lepidoptera: Pyralidae) et de leurs hybrides. Agronomie 2, 989-994

Stephan E (1989) Validation du modèle de durée de développement des stades larvaires de la pyrale du maïs (Ostrinia nubilalis Hübner (Lepidoptera: Pyralidae) dans les conditions environnementales de Bordeaux. Mémoire de DAA, spécialité "Protection des cultures", INA-PG, ENSAM, ENSAR, ENSAA, $20 \mathrm{p}$

Stinner RE, Butler GD, Bacheler JS, Tuttle C (1975) Simulation of temperature-dependent development in population dynamics models. Can Entomol 107, 1167-1174

Wada T, Kobayashi M (1980) Effects of temperature on development of the rice leaf roller, Cnaphalocrocis medinalis Guenée (Lepidoptera: Pyralidae). Appl Entomol Zool 15, 207-214

Wagner TL, Hsin-I Wu, Sharpe PJH, Schoolfield RM, Coulson RN (1984a) Modeling insect development rates: a literature review and application of a biophysical model. Ann Entomol Soc Am 77, 208-225

Wagner TL, Hsin-I Wu, Sharpe PJH, Coulson RN (1984b) Modeling distributions of insect development time: a literature review and application of the weibull function. Ann Entomol Soc Am 77, 475-487 